\title{
Flexibility as a marker of early cognitive decline in humanized Apolipoprotein E \&4 (ApoE4) mice.
}

Abbreviated title (50 characters max): Early flexibility deficit in ApoE4 mice

Schmitt Julien ${ }^{1}$ 2, Paradis Anne-Lise ${ }^{1}$, Boucher Mathieu ${ }^{3}$, Andrieu Laurent ${ }^{4}$, Barnéoud Pascal $^{2} \&$ Rondi-Reig Laure ${ }^{1 \S}$.

${ }^{1}$ Sorbonne Université, CNRS, INSERM, Institut de Biologie Paris Seine (IBPS), Neurosciences Paris Seine (NPS), Cerebellum Navigation and Memory Team (CeZaMe), F75005 Paris, France

${ }^{2}$ Neurodegeneration Cluster, Rare \& Neurologic Diseases Research, Sanofi R\&D, F-91380 Chilly-Mazarin, France

${ }^{3}$ IT\&M STATS, F-92200 Neuilly sur Seine, France

${ }^{4}$ Biostatistics \& Programming Department, Non-Clinical Efficacy \& Safety team, Sanofi R\&D, F-94400 Vitry-Sur-Seine

§To whom correspondence should be addressed. E-mail: laure.rondi-reig@upmc.fr IBPS, Neurosciences Paris Seine, Sorbonne Université, UPMC Univ Paris 06, CeZaMe team, 9 Quai Saint Bernard, 5e étage, 75005 Paris, France

\section{Author Contributions:}

Financial support: PB, LRR

Designed the project and supervised the PhD work: LRR

Designed experiments: JS, LRR

Performed experiments: JS

Designed analysis and analyzed data: JS, LRR, ALP, MB, LA

Wrote the paper: all the co-authors

\section{Abbreviations:}

ApoE: apolipoprotein $\mathrm{E}$

AD: Alzheimer's disease

$\mathrm{MCl}$ : Mild Cognitive Impairment

$A \beta$ : beta-amyloid

RPM: rotations per minute

GLMM: generalized linear mixed model

ANOVA: Analysis of variance

MAD: median absolute deviation

SEM: standard errors of mean

WSCT: Wisconsin Sorting Card Test

VS.: versus 


\section{Highlights}

- ApoE4 mice tested with spatial reversal learning present early flexibility deficit

- This flexibility deficit is not associated with memory impairment

- ApoE4 appears as a factor of early aging

\section{Abstract (145/170 words max)}

To test the hypothesis that ApoE4 may be involved in cognitive deficits associated with aging, we investigated the impact of APOE4 status and aging on the flexibility and memory components of spatial learning in mice. Young adult (6 months) and middle-aged (14 months) ApoE4, ApoE3 and C57BL/6 adult male mice were tested for flexibility in an aquatic $\mathrm{Y}$-maze, and for spatio-temporal memory acquisition in the Starmaze. Our results revealed a flexibility deficit of the 6-month-old ApoE4 mice compared to controls. However, this deficit was not associated with spatio-temporal memory deficit at the same age. Importantly, the ApoE4 flexibility deficit did not increase with age, nor turn into memory deficit, or was able to predict individual variations of memory performance at 14 months. By contrast, control ApoE3 mice showed a decline of flexibility at 14 months resulting in performance similar to that of ApoE4. Overall, our results suggest that ApoE4 could be associated with an acceleration of the flexibility decrease otherwise observed in normal aging. 


\section{Keywords}

ApoE4; Flexibility; Reversal Learning; Spatio-temporal memory; Aging; Navigation behavior. 


\section{Introduction}

The E4 isoform of apolipoprotein E is currently considered to be the main genetic risk factor associated with the sporadic form of Alzheimer's disease (AD). Indeed, while the distribution frequency of allele $\varepsilon 4$ is $13 \%$ in a control population, it reaches about $30 \%$ in the population with AD (Farrer et al., 1997). A person homozygous for APOE$\varepsilon 4$ is about 13.7 times more likely to develop the disease than a person homozygous for APOE- $\varepsilon 3$, and for APOE $\varepsilon 3 / \varepsilon 4$ heterozygotes, the risk is about three times higher. These findings, and specifically the dose-related association, have led to the idea that the E4 isoform is involved in late-onset $A D$.

Interestingly, studies have also reported a decline in cognitive functions such as processing speed and memory (Caselli et al., 2009; Dik et al., 2001; Rawle et al., 2018; Zehnder et al., 2009) as well as executive functions (Allain et al., 2013, for review) in aged APOE- $\varepsilon 4$ carriers, independently of mild cognitive impairment $(\mathrm{MCl})$ or dementia diagnostic. An impairment in inhibition/switching tasks was also observed in aged nonAD APOE- $\varepsilon 4$ carriers (Chao et al., 2010; Wetter et al., 2005). Altogether, these results strongly point toward a detrimental effect of carrying the $\varepsilon 4$ allele in aged people. In addition, a longitudinal study between ages 11 and 80 found significant cognitive differences depending on the ApoE4 status (homozygous, heterozygous or non-carrier) at age 80 but not 11, suggesting ApoE4 could be a factor of cognitive decline with normal aging (Deary et al., 2002). However, this effect appears independent of the $A D$ or $\mathrm{MCl}$ diagnostic, thus questioning the link between the observed cognitive deficits, the aging process per se and Alzheimer's disease. 
Behavioral studies with knock-in mouse models expressing the human ApoE3 (Sullivan et al., 1997) and ApoE4 (Knouff et al., 1999) variants showed seemingly contradictory results on spatial learning and memory assessment depending on the task used (Rodriguez et al., 2013). Rodriguez et al. (2013) found a learning deficit for ApoE4 mice in the Barnes maze but not in the Morris water maze, suggesting that different cognitive components, such as spatial memory acquisition or flexibility, may be differently involved in these tasks. In addition, different types of flexibility may also be involved. Indeed, Brown and Tait (2010) defined two categories of flexibility tasks, either based on mental set shifting (attentional set or learning set) or reversal learning (reversal of stimulus-reward contingency), the former being more often used in humans and the latter in mice.

We hypothesize that such cognitive components might be differently impaired with APOE4 status. To address this question, we tested the ability of ApoE4 mice to both acquire spatio-temporal memory and perform a flexibility test based on reversal learning. Spatio-temporal memory was assessed using a previously developed navigation task, the Starmaze (Rondi-Reig et al., 2005). This Starmaze allowed the detection of early deficits in mice (Fouquet et al., 2011) and humans (Bellassen et al., 2012), and could disentangle normal aging from AD-specific deficits in humans (Bellassen et al., 2012). As a reversal learning test, we used the aquatic Y-maze (Hoeffer et al., 2008; Trinh et al., 2012). In order to assess possible interactions between APOE4 status, aging, flexibility and spatiotemporal memory, we tested these two cognitive functions in parallel in both young adult (6 months) and middle-aged (14 months) APOE4, APOE3 and C57BL/6 male mice. 
Altogether, our results suggest that ApoE4 could be associated with an early apparition of flexibility alteration otherwise observed during normal aging in mice.

\section{Material and Methods}

\subsection{Animals}

All experiments were approved by Sorbonne University Ethical Committee and conducted in full compliance with standards for the care and use of laboratory animals, according to French and European Community (Directive 2010/63/EU) legislation. Animals were housed in a 12 hours dark/light cycle in a temperature-controlled room (20 $+/-1^{\circ} \mathrm{C}$ ) with food and water ad libitum. They were housed by groups of 3 or 4 mice per cage until one week before the start of the experiment, at which point mice were isolated in individual cages until the end of the first experiment, which lasted approximately 1 month.

Knock-in male ApoE3 and ApoE4 mice were obtained from Taconic Biosciences (Germantown, USA). These mice are homozygous for human APOE3 and APOE4 variants expressed under the control of the murine ApoE regulatory sequences. A first group of ApoE3 $(n=14)$ and ApoE4 $(n=14)$ mice was tested at 6 months. A second group was specifically used for a longitudinal study with ApoE3 $(n=15)$ and ApoE4 $(n=13)$ mice tested at 6 months then at 14 months of age to evaluate the evolution of their cognitive abilities independently of old-age. After the first experiment, mice for the longitudinal study were housed two per cage until the second experiment at 14 months old. The number of mice used for each protocol is indicated in the results section. 
C57BL/6JRj male mice aged 6 months $(n=15)$ were obtained from Janvier Labs (Saint Berthevin, France) to be used as controls for the first group of 6-month-old ApoE3 and ApoE4.

\subsection{Behavioral tests}

\subsubsection{Sequence of the tests}

All mice performed the tests described below in the following order: Shirpa protocol (inspired by Rogers et al., 1997, see supplementary material), Starmaze, aquatic Y-maze and cued Morris water maze (performed to ensure that possible deficits observed during the Starmaze task are not due to vision or swimming deficits).

\subsubsection{Starmaze}

The Starmaze paradigm is used to assess spatio-temporal memory. The Starmaze is a star-shaped water maze consisting of 5 central alleys forming a pentagon and 5 peripheral alleys starting from each vertex of the pentagon. In order to reduce the acquisition period, we here used a reduced Starmaze: some alleys were made inaccessible by blocking them with walls (see Fig. 2E). The alleys are 42 (central) or 47 (peripheral) cm long, $25 \mathrm{~cm}$ wide and $30 \mathrm{~cm}$ high (Rondi-Reig et al., 2005). The Starmaze is filled with water made opaque with a non-toxic dye (Accuscan OP 301) up to about $5 \mathrm{~cm}$ from the top of the walls. A platform is immersed $1 \mathrm{~cm}$ below the water surface. The Starmaze is surrounded by black curtains on which 2D or 3D visual cues are attached. The cues are grouped into groups of 2 or 3 . All cues are present in two copies but each configuration of 2 or 3 cues is unique. 
A trial begins with the mouse released by the experimenter always at the same departure point. The mouse is then left to swim until it finds the platform or 90 seconds maximum. If the mouse finds the platform, it is left 20 seconds on the platform before being picked up by the experimenter. If the mouse does not find the platform before the end of the 90 seconds, it is placed on the platform for 20 seconds. At the end of each trial, the mouse is placed in a cage containing a towel for drying before being returned to its home cage while waiting for the next trial, during 30 to 60 minutes. The acquisition phase consisted of 5 trials per day for 8 days.

For the mice tested twice, at 6 and 14 months, the environment did not change. The experiment took place in the same room with the same arrangement of visual cues around the Starmaze. The start location was the same but the arrival location changed so that the sequence to reach it mirrored that of the previous learning, i.e mice should turn right-left-right rather than left-right-left. For this purpose, the arrangement of the blocking walls was also adapted.

After testing their robustness (see associated Schmitt et al., Data in brief), four behavioral parameters were used in this study $: 1)$ The distance travelled during a trial, measured by the tracking software (SMART 2.5, Bioseb); 2) The localization score, calculated by allocating a mark at each choice point (100 if the mouse moves toward the platform, 0 otherwise) and averaging the marks allocated at all the intersections encountered during a trial (Fouquet et al., 2011); 3) The repeated sequence score, attributing a maximal mark if the moves made at each choice point of trial Ti+1 reproduce the choices made at trial Ti, in a same learning session (Bellassen et al., 2012); 4) The number of dead ends (peripheral arms not containing the platform) revisits. 
To further evaluate possible correlations between the flexibility performances in the Y-maze and memory acquisition in the Starmaze, we also considered two learning indexes consisting of the difference (hereafter called Delta) between the last and first trials in the Starmaze for the travelled distance and the localization score.

We also quantified the search strategies used by the different groups of mice during the acquisition of the task. We thus identified two types of strategy: direct (efficient sequence of turns to the goal with no visit of dead ends), and serial strategy (systematic visit of the dead end between the start and the platform). Other types of behavior were labelled as "no strategy".

\subsubsection{Aquatic Y-maze}

Cognitive flexibility was assessed using an aquatic Y-maze test. This Y-maze consists of 3 opaque arms $50 \mathrm{~cm}$ long, $10 \mathrm{~cm}$ wide and $30 \mathrm{~cm}$ high. It is filled with $12 \mathrm{~cm}$ of water made opaque by a non-toxic colorant (Accuscan OP 301). A circular platform (9 $\mathrm{cm}$ in diameter) is placed $1 \mathrm{~cm}$ below the water surface at the end of one arm. The protocol, based on the one described by Hoeffer et al. (2008), lasts 2 days with the four sessions of the learning phase on day 1 and the test and the six sessions of reversal on day 2.

For the mice tested twice, at 6 and 14 months, the environment of testing did not change. The test took place in the same room with the same black curtains surrounding the maze to avoid visual cues. The only change was the place of the platform in the Ymaze during the learning phase: at 14 months, the platform was placed in the opposite arm from the location used at 6 months. 


\subsection{Acquisition and Analysis of raw data}

Each mouse's trajectory was tracked in the Starmaze, the Morris water maze and several sensorimotor tests using SMART 2.5 (Bioseb) software. We thus recorded the mouse coordinates ( $\mathrm{X}$ and $\mathrm{Y}$ ) every 0.04 seconds. These coordinates were then processed with our own developed software, NAT (Navigation Analysis Tool (Jarlier et al., 2013)), to compute the assessment scores presented below.

\subsection{Statistical analysis}

All statistical analyses were performed to compare the effect of the mouse genotype on cognition through several parameters. The number of animals per group was based on the availability of mice with these genotypes, and prior studies with other strains (Fouquet et al., 2011). We used SAS 9.4 (SAS Institute Inc., Cary, NC, USA) on Windows 7 PC.

For the percentages of success into the aquatic $Y$-maze with 6 month-old mice, two (one for the learning phase, one for the reversal phase) binomial generalized linear mixed models (GLMM) were performed on factors Genotype and Session (repeated) followed by a contrast analysis using a Student's T test with Bonferroni-Holm correction for multiplicity (Table 1). For the subsequent longitudinal study, two GLMM using binomial distribution were performed with the three factors Genotype, Age (repeated) and Session (repeated) (Table 2).

When comparing ApoE3, ApoE4 and C57BL/6 at 6 months in the Starmaze, the distance travelled was analyzed using a two-way analysis of variance (ANOVA) with factors Genotype, Session and their interaction performed on log-transformed data. 
Repeated measures on the factor session were taken into account in each model and $A R(1)$ variance-covariance structure was used. The Localization score was analyzed using two-way ANOVA with factors Genotype, Session and their interaction performed on raw data. For the other two Starmaze parameters, i.e. repeated sequence score and Dead end revisit number, a two way ANOVA-type was performed on ranked data. To compare the use of search strategies between groups, a two-way GLMM with binomial distribution was performed on raw data for the number of Direct, Serial and No strategy trials (Table 3).

To test for possible correlations between the performances in the Y-maze at 6 months and the Starmaze at 6 and 14 months, in the longitudinal group, a Spearman's rank correlation test was performed (Table 4).

\subsection{Code Accessibility}

NAT (Navigation Analysis Tool) code is protected by the Inter Deposit Digital Number FR.001.160024.000.S.P.2017.000.10000, 2017 


\section{Results}

\subsection{Aquatic Y-maze}

3.1.1. Six-month-old ApoE4 mice exhibit cognitive flexibility deficit

To assess cognitive flexibility in 6-month-old ApoE4 mice, we compared the performances of ApoE4 mice with those of ApoE3 and C57BL/6 mice of the same age in the Y-maze. In ApoE3 ( $n=14)$ and C57BL/6 $(n=15)$ groups, one mouse did not reach the criterion in the test session, so only $13 \mathrm{ApoE} 3$ and $14 \mathrm{C} 57 \mathrm{BL} / 6$ mice were considered in the analysis. In the ApoE4 ( $n=14)$ group, two mice did not reach the criterion, so only 12 mice were considered.

During the learning phase, the performances of 6-month-old ApoE4, ApoE3 and C57BL/6 improved with sessions (Session effect: $F(3,107.9)=11.51, p<0.0001$, binomial GLMM) (Fig. 1A left). Compared to ApoE3 and C57BL/6 mice, ApoE4 mice had less success in session 2 (Genotype effect: $F(2,52.71)=4.56, p=0.0149$, binomial GLMM; ApoE4 vs ApoE3 S2: $p=0.0090$, ApoE4 vs C57BL/6 S2: $p=0.0237$, after Bonferroni-Holm correction for multiplicity). Importantly, there was no significant difference between ApoE4 and ApoE3 or C57BL/6 mice at the end of the learning ( $p>0.05$ for each comparison in S3 and S4) (Fig. 1A left).

During reversal sessions, the three groups improved their performance (Session effect: $F(5,189.3)=24.07, p<0.0001$, binomial GLMM). However, ApoE4 mice had a significantly lower success rate during reversal sessions than ApoE3 and C57BL/6, owing to differences in sessions 2, 3 and 4 (Genotype effect: $F(2,51.17)=6.32, p=0.0035$, binomial GLMM; ApoE4 vs ApoE3 S2: $p=0.0154$, S3: $p=0.0026, S 4: p=0.0374$, ApoE4 vs C57BL/6 S2: $p=0.0227$, S3: $p=0.0026$, S4: $p=0.0374$, Bonferroni-Holm adjustment after 
binomial GLMM) (Fig. 1.A right, see statistical results in Table 1). These results suggest a cognitive flexibility deficit of the whole group of ApoE4 mice.

We further controlled whether sensory-motor differences in the control tests (SHIRPA protocol) may explain the flexibility deficit observed here. The comparison between the 6-month-old C57BL/6, ApoE3 and ApoE4 mice only revealed that ApoE3 mice seemed to travel less distance in the open field than both C57BL/6 and ApoE4 mice (see supplementary data). Since the smaller distance travelled by ApoE3 mice does not translate into greater flexibility performance compared to C57BI6, it is unlikely that sensory-motor differences explain the flexibility deficit observed in the ApoE4 mice.

3.1.2. The cognitive flexibility of 14 month-old ApoE3 mice declines at the level of 6-monthold ApoE4 mice

To test if the observed early flexibility deficit evolves with age, we tested two other groups of ApoE4 and ApoE3 mice both at 6 and 14 months in the Y-maze. For this longitudinal study, only the performances of the mice who underwent all the sessions at both 6 and 14 months were considered, i.e., 14 ApoE3 and 9 ApoE4 mice. During the learning phase of the aquatic Y-maze, whatever their age, ApoE3 and ApoE4 mice increased their success rate (Session effect: $F(3,168)=22.35, p<0.0001$, three-way repeated measures binomial GLMM) and there was no difference of performance between the two groups (Genotype effect: $F(1,25.26)=0.42, p=0.5250$, three-way repeated measures GLMM) (Fig. 1B left, Table 2). Aging had no impact on the learning performance of any group of mice (Age*Genotype effect: $F(1,25.03)=0.16, p=0.6921$, three-way repeated measures GLMM). 
In the reversal phase, performances increased with sessions (Session effect: $F(5$, $99.63)=47.56, p<0.0001$, three-way repeated measures GLMM). However, we observed an age* genotype interaction effect $(F(1,21.56)=4.38, p=0.0483$, three-way repeated measures GLMM). Post hoc analyses reveal that ApoE3 group performance significantly decreases with age in session 2 and session 4 (S2: ApoE3 6 months vs ApoE3 14 months, $p=0.0081 ;$ S4: ApoE3 6 months vs ApoE3 14 months, $p=0.0342$ ) while age does not impact ApoE4 performance which remains similar at 6 and 14 months (Fig. 1B right, Table 2). It is noteworthy that session by session comparisons between ApoE4 and ApoE3 mice at 6 months confirm, with the subgroups of this longitudinal study, the ApoE4 deficit observed with the first 6 month groups in the reversal phase (Genotype effect at S2: p0.0001, S3: $p=0.0096$, bottom of Table 2). Those results suggest that ApoE4 could be an aging accelerator factor of cognitive flexibility. The representation of the individual performances of the mice at 6 then 14 months in sessions 2 to 4 illustrates the decrease of ApoE3 performances and the stability of the ApoE4 performances (Fig. 1.C).

\subsection{Starmaze}

To evaluate if the flexibility deficit observed at 6 months has a counterpart in the spatial learning, we assessed the ability of the ApoE4 mice tested in the Y-maze to acquire the Starmaze task.

The Starmaze offers the possibility to compute multiple scores, either generic to this environment (Jarlier et al., 2013) or specific to its reduced version (with closed arms). We tested four behavioral parameters (see methods) previously revealed to be specific for different functions: the travelled distance, reflecting the learning of the task through 
its evolution across sessions; the localization score, associated with spatial memory (Fouquet et al., 2011); the repeated sequence score, assessing the memory of sequence (Bellassen et al., 2012) inside sessions; and the number of dead end revisits, likely associated with working memory (Beatty et al., 1984).

3.2.1. ApoE4 mice do not exhibit memory deficits in the Starmaze task.

We compared the memory performances of the 6 month-old C57BL/6, ApoE3 and ApoE4. In the four scores of the Starmaze (travelled distance -TD-, localization score -LS-, repeated sequence score -RSS- and dead end revisit number -DERN-), the three groups increased their performances with sessions (TD: $F(7,266.2)=52.12, p<0.0001 ; L S: F(7$, $264)=27.90, p<0.0001 ;$ RSS: $F(3.39,195)=6.60, p<0.0001 ; \operatorname{DERN}: F(6.48,319)=27.34$, $p<0.0001$; Fig. 2A, B, C and D). We did not observe any significant difference of performances between the three groups nor interaction between group and session (Table 3), suggesting that 6 month-old ApoE4 mice do not exhibit memory deficit in this Starmaze task associated with their flexibility deficit. The analysis of the search strategy in the Starmaze (Fig 2. E) confirmed the absence of deficit in ApoE4 mice compared to both control groups (Table 3).

3.2.2. No link between early flexibility deficit and spatio-temporal memory with aging

In the longitudinal group, we tested whether the flexibility deficit observed in APoE4 mice at 6 months may be linked to later memory impairments at the individual level. We therefore tested possible correlations between the performance during reversal sessions S2 and S3 (flexibility index) in the aquatic Y-maze at 6 months and two learning indexes in the Starmaze at 14 months (Fig. 3, Table 4). The correlations were performed 
at the individual level in each group separately. In the ApoE4 group, there was no significant correlation between the flexibility index and the learning indexes in the Starmaze (Delta travelled distance, $r h o=-0.214, p=0.5455$; Delta localization score, rho $=-0.591, p=0.0944$, Spearman's rank correlation). The correlations were not significant in the ApoE3 group either (Delta travelled distance: rho $=0.219$; Delta localization score: rho $=0.1228$, Spearman's rank correlations). We further controlled in these groups that there was no significant correlation between the flexibility and learning indexes at 6 months (ApoE4 - Delta TD: rho $=-0.316$; Delta LS: rho $=-0.021, p=0.9516 ;$ ApoE3 - Delta TD: rho $=0.111 ;$ Delta LS: rho $=0.418$, Spearman's rank correlations, Fig. 4). Thus, the flexibility deficit observed in the Y-maze for ApoE4 mice at 6 months was not associated with any impairment in the acquisition of the Starmaze task either at 6 or 14 months.

Overall, the deficit of flexibility observed at 6 months in the ApoE4 mice expressing the major risk factor of the sporadic form of Alzheimer's disease was not associated with learning deficits at the same age nor predict individual variations of learning performances at 14 months.

\section{Discussion}

Our results show that 6-month-old ApoE4 male mice exhibit a flexibility deficit compared to ApoE3 and C57BL/6 control mice at the same age. This flexibility deficit is not associated with memory impairment. In addition, the ApoE4 deficit does not significantly evolve with age, while ApoE3 mice at 14 months display a slight flexibility 
decrease, leading to similar performances across the 14-month-old groups. These results highlight the impact of the ApoE4 status on flexibility regardless of age.

\subsection{ApoE4, aging and cognitive flexibility.}

Few studies have explored the impact of ApoE4 status on flexibility, and even less have examined the link between cognitive flexibility, ApoE4 and aging. Lancaster et al. (2017) conducted a meta-analysis on studies involving adult ApoE4 subjects (35 to 60 years of age). In this age group, only subtle effects of ApoE4 on memory and executive functions were observed, but with no specific focus on cognitive flexibility. The authors also emphasized the importance of stratifying by age, because of the potential agerelated confounding effect. Adeosun et al. (2014) observed a deficit in cognitive flexibility in 12 months old Arg-61 mice, a model of ApoE4 domain interaction. It is noteworthy that our results highlight a clear lack of flexibility in homozygous E4 animals as early as 6 months, revealing the deleterious effect of ApoE4 even at a young age.

By contrast, our ApoE3 mice showed a significant loss of flexibility with aging. In line with this, Yang et al. (2019) found a flexibility loss with normal aging using a Y-maze test and comparing C57BL/6 male mice at 4 and 20 months. Matzel et al. (2011), using a T-maze test with two groups of 5 and 18-month-old CD-1 male mice also found a significant deficit of flexibility in the older group, as well as a longer time to learn the initial task, similar to what can be observed with our 6 month-old apoE4 mice in the learning phase (Fig. 1.A left). Interestingly, similar effects of normal aging on cognitive flexibility have also been found in other species, such as rats (Zamzow et al., 2013; Schoenbaum et al., 2002 and Barense et al., 2002), monkeys (Joly et al., 2014) and humans (An et al., 2018; Boxtel et al., 1998; Stöckel et al., 2017). The early flexibility deficit we observed in ApoE4 
is therefore consistent with the advanced onset of a flexibility decrease otherwise observed with normal aging.

It is noteworthy that when testing flexibility during aging, contradictory findings have been reported, especially when the flexibility assessment relied on attentional set shifting rather than reversal learning. Zeamer et al. (2011) did not find any effect of age when testing male macaques with an adapted version of the Wisconsin Sorting Card Test (WSCT), but did find age-related deficits in visual discrimination reversal. Hankosky et al. (2017) did not observe any flexibility deficit in 18-month-old rats in a set-shifting test requiring changing strategy (from the use of visual cues to egocentric orientation). Beas et al. (2013) reported flexibility deficits with the same task but the age difference was also much larger ( 5 vs. 22 months instead of 12 vs. 18 months). Together with our results, this suggests that cognitive evaluation would be improved by systematically including a flexibility test based on reversal learning contingency.

The type of test used to assess flexibility is also of particular importance since reversal learning and attentional shifting involve different brain structures. In a a odordiscrimination task with aged rats, Schoenbaum et al. (2002) reported reversal learning deficit, reminiscent of the deficit observed in young rats following neurotoxic lesions of orbitofrontal cortex (OFC). By contrast, reversal learning is not affected following mPFC damage in rodents (Birrell and Brown, 2000, Bissonette et al., 2008; Floresco et al., 2008; Churchwell et al., 2009; Cordova et al., 2014). Rather, it has been proposed that mPFC may be "primarily involved in tasks with a high demand on attention" such as attentional set-shifting tasks (Barense et al., 2002). Altogether these data point toward a distinct role of $\mathrm{mPFC}$ and OFC in attentional set-shifting task and reversal learning respectively. The 
type of test used to assess executive functions therefore appears crucial to obtain consistent results and pleads for generalizing the use of flexibility tests based on reversal learning contingency in the screening of APoE4 carriers.

\subsection{Acquisition of spatio-temporal memory in mouse models of risk of $A D$}

As mentioned in the introduction, published data in ApoE4 mice show heterogeneous results in memory tasks, likely depending on age, sex and the test performed to assess memory. Here, despite an early flexibility deficit observed in the $\mathrm{Y}$ maze, ApoE4 male mice do not show any learning deficit at 6 or 14 months in the Starmaze, consistent with previous studies suggesting that ApoE4 males, unlike females, do not develop spatial memory deficits (Adeosun et al., 2019; Grootendorst et al., 2005; Reverte et al., 2012; Ungar et al., 2014).

Could the absence of deficit at 14 months be explained by the previous experience of the mice with the starmaze environment at 6 months? Although the 14-month-old mice already knew the environment and the task, the sequence of turns to reach the platform was different (mirrored sequence compared to the 6 month one) and thus had to be learned again. Accordingly, the learning indexes clearly demonstrate a new learning in all groups (Fig. 3). In addition, that 14-month-old APPPS1 mice, modeling the familial form of $A D$, show a clear deficit of spatio-temporal learning (see associated Schmitt et al., Data in Brief) consistent with the expected development of an AD pathology in these mice (Blanchard et al., 2003), rules out a possible lack of sensitivity in our test. Therefore, the Starmaze task being hippocampus dependent (Rondi-Reig et al., 2006; Fouquet et al., 
2013), our results suggest that this hippocampal-dependent memory, as tested in the Starmaze, remained intact in the ApoE4 risk model mice.

\subsection{Is there a link between Flexibility and Spatio-temporal memory in ApoE4 mice on an individual basis?}

Although no memory deficit was observed at the group level, we could not exclude that ApoE4 mice, as a model of risk factor only, may display individual variability in their ability to acquire spatio-temporal memory. To take this possibility into account, we tested the correlation between the success rate in the reversal phase of the Y-maze at 6 months and two learning indexes in the Starmaze at both 6 and 14 months. Our results show that the early flexibility deficit observed at 6 months was not associated either with memory acquisition deficit at the same age or with the subsequent development of memory impairments. Thus, in ApoE4 mice both cognitive functions appear independent with no cross-effect during aging, consistent with Adeosun et al. hypotheses (Adeosun et al., 2014). The fact that we do not observe correlation in ApoE3 either suggests that these two functions are independent and differentially altered in normal aging as well.

Although we did not find any correlation between the two behavioral parameters, it is noteworthy that only 9 ApoE4 mice (vs. 14 ApoE3 and C57BL/6 mice) could be tested in the longitudinal flexibility study. Indeed 4 mice were excluded for having failed the learning phase of the Y-maze protocol at 6 months $(n=1), 14$ months $(n=1)$ or both $(n=2)$. This larger number of exclusions in the ApoE4 group might reveal a specific learning deficit. However, examining the global learning performances of these excluded mice in the Starmaze (localization score and travelled distance across the last 5 sessions at 6 and 
14 months) shows that they globally rank in the middle of the distribution (between quartiles 2-3): this is in particular the case for the 2 mice having failed both Y-maze sessions; some Starmaze values were found in the lowest quartile: this is the case for the performance at 6 months of the mouse that failed the $Y$-maze learning at 14 months, and performances at 14 months for the mouse that failed the Y-maze at 6 months.

Overall, our results show that ApoE4-related deficits target reversal flexibility independently of hippocampus-dependent memory.

\section{Conclusion: ApoE4, a factor of early aging rather than cognitive}

\section{decline with aging}

Since ApoE4 is associated with an enhanced risk of developing Alzheimer's disease, especially in the case of homozygosity, memory deficits were expected to be found in this model. In our study, however, aging ApoE4 did not show any spatio-temporal memory deficits, in contrast to APPPS1 mice of the same age, a model of the familial form of Alzheimer's disease. By targeting males, this study rather revealed a flexibility deficit independent of any memory acquisition impairment. In addition, the ApoE4 mice did not present increased decline with aging, but displayed an early flexibility deficit stable with age. The risk of developing AD in Apoe4 homozygosity could be related to this early aging. Overall, our results reveal that the ApoE4 status could be associated with an acceleration in the appearance of flexibility alteration usually observed during normal aging in mice. 


\section{Acknowledgements}

This work was supported by Sanofi (PB, JS, LA), the CNRS and Sorbonne University through UMR 8246 (LRR). The group of LRR is a member of the Labex BioPsy and ENP Foundation. This work also received support under the program Investissements d'Avenir launched by the French Government and implemented by the ANR, with the references ANR-10-LABX-BioPsy (LRR). Labex are supported by French State funds managed by the ANR within the Investissements d'Avenir programme under reference ANR-11-IDEX-000402. We thank Jean Vincent for help with behavioral experiments. We thank Magali Maroccia for supplementary statistical analyses. We thank Chantal Mathis and Nora Abrous for their scientific insight on the manuscript and Mati Lopez-Grancha, Elisabeth Genet, Nicolas Moindrot and Philippe Goniot for fruitful discussions. We thank the IBPS animal facility for helping in taking care of mice.

\section{Disclosure}

JS, PB and LA were full-time employees of Sanofi when this study was performed. The CNRS, LRR and her lab have financial interest in the sale of the Starmaze task.

\section{References}

Adeosun, S.O., Hou, X., Shi, L., Stockmeier, C.A., Zheng, B., Raffai, R.L., Weisgraber, K.H., Mosley, T.H., Wang, J.M., 2019. Female mice with apolipoprotein E4 domain interaction demonstrated impairments in spatial learning and memory performance and disruption 
of hippocampal cyto-architecture. Neurobiol. Learn. Mem. 161, 106-114. https://doi.org/10.1016/j.nlm.2019.03.012

Allain, P., Etcharry-Bouyx, F., Verny, C., 2013. Executive functions in clinical and preclinical Alzheimer's disease. Rev. Neurol. (Paris). 169, 695-708. https://doi.org/10.1016/j.neurol.2013.07.020

An, Y., Feng, L., Zhang, X., Wang, Ying, Wang, Yushan, Tao, L., Lu, Y., Qin, Z., Xiao, R., 2018. Patterns of cognitive function in middle-aged and elderly Chinese adults-findings from the EMCOA study. Alzheimers. Res. Ther. 10, 93. https://doi.org/10.1186/s13195-018$0421-8$

Barense, M. D., Fox, M. T., \& Baxter, M. G., 2002. Aged rats are impaired on an attentional set-shifting task sensitive to medial frontal cortex damage in young rats. Learning and Memory, 9(4), 191-201. https://doi.org/10.1101/Im.48602

Beas, B.S., Setlow, B., Bizon, J.L., 2013. Distinct manifestations of executive dysfunction in aged rats. Neurobiol. Aging 34, 2164-2174.

https://doi.org/10.1016/J.NEUROBIOLAGING.2013.03.019

Beatty, W.W., Bierley, R.A., Boyd, J., 1984. Amphetamine disrupts both working and reference memories of rats trained in a radial maze. Behav. Neural Biol. 42, 169-76.

Bellassen, V., Igloi, K., de Souza, L.C., Dubois, B., Rondi-Reig, L., 2012. Temporal Order Memory Assessed during Spatiotemporal Navigation As a Behavioral Cognitive Marker for Differential Alzheimer's Disease Diagnosis. J. Neurosci. 32, 1942-1952. https://doi.org/10.1523/JNEUROSCI.4556-11.2012

Bissonette, G. B., Martins, G. J., Franz, T. M., Harper, E. S., Schoenbaum, G., \& Powell, E. M., 2008. Double dissociation of the effects of medial and orbital prefrontal cortical lesions 
on attentional and affective shifts in mice. Journal of Neuroscience, 28(44), 11124-11130. https://doi.org/10.1523/JNEUROSCI.2820-08.2008

Blanchard, V., Moussaoui, S., Czech, C., Touchet, N., Bonici, B., Planche, M., Canton, T., Jedidi, I., Gohin, M., Wirths, O., Bayer, T.A., Langui, D., Duyckaerts, C., Tremp, G., Pradier, L., 2003. Time sequence of maturation of dystrophic neurites associated with $A \beta$ deposits in APP/PS1 transgenic mice. Exp. Neurol. 184, 247-263. https://doi.org/10.1016/S00144886(03)00252-8

Boxtel, M.P.J. v., Buntinx, F., Houx, P.J., Metsemakers, J.F.M., Knottnerus, A., Jolles, J., 1998. The Relation Between Morbidity and Cognitive Performance in a Normal Aging Population. Journals Gerontol. Ser. A Biol. Sci. Med. Sci. 53A, M147-M154. https://doi.org/10.1093/gerona/53A.2.M147

Brown, V.J., Tait, D.S., 2010. Behavioral Flexibility: Attentional Shifting, Rule Switching and Response Reversal, in: Stolerman, I.P. (Ed.), Encyclopedia of Psychopharmacology. Springer Berlin Heidelberg, Berlin, Heidelberg, pp. 209-213. https://doi.org/10.1007/9783-540-68706-1_340

Caselli, R.J., Dueck, A.C., Osborne, D., Sabbagh, M.N., Connor, D.J., Ahern, G.L., Baxter, L.C., Rapcsak, S.Z., Shi, J., Woodruff, B.K., Locke, D.E.C., Snyder, C.H., Alexander, G.E., Rademakers, R., Reiman, E.M., 2009. Longitudinal Modeling of Age-Related Memory Decline and the APOE $\varepsilon 4$ Effect. N. Engl. J. Med. 361, 255-263. https://doi.org/10.1056/NEJMoa0809437

Chao, L.L., Kramer, J.H., Miller, B.L., Yaffe, K., Madison, C., Weiner, M.W., 2010. Deficits in cognitive flexibility are associated with APOE-E4 allele and precuneus volume and thickness in nondemented older adults. Alzheimer's Dement. 6, S441-S442. 
https://doi.org/10.1016/J.JALZ.2010.05.1471

Churchwell, J. C., Morris, A. M., Heurtelou, N. M., \& Kesner, R. P., 2009. Interactions Between the Prefrontal Cortex and Amygdala During Delay Discounting and Reversal. Behavioral Neuroscience, 123(6), 1185-1196. https://doi.org/10.1037/a0017734

Cordova, C. A., Jackson, D., Langdon, K. D., Hewlett, K. A., \& Corbett, D., 2014. Impaired executive function following ischemic stroke in the rat medial prefrontal cortex. Behavioural Brain Research, 258, 106-111. https://doi.org/10.1016/j.bbr.2013.10.022

Deary, I.J., Whiteman, M.C., Pattie, A., Starr, J.M., Hayward, C., Wright, A.F., Carothers, A., Whalley, L.J., 2002. Cognitive change and the APOE \&4 allele. Nature 418, 932-932. https://doi.org/10.1038/418932a

Dik, M.G., Jonker, C., Comijs, H.C., Bouter, L.M., Twisk, J.W.R., Kamp, G.J. van, Deeg, D.J.H., 2001. Memory complaints and APOE-epsilon4 accelerate cognitive decline in cognitively normal elderly. Neurology 54, 1492-1497. https://doi.org/10.1212/wnl.57.12.2217

Farrer, L.A., Cupples, L.A., Haines, J.L., Hyman, B., Kukull, W.A., Mayeux, R., Myers, R.H., Pericak-Vance, M.A., Risch, N., Duijn, C.M. van, 1997. Effects of Age, Sex, and Ethnicity on the Association Between Apolipoprotein E Genotype and Alzheimer Disease. JAMA 278, 1349. https://doi.org/10.1001/jama.1997.03550160069041

Floresco, S. B., St. Onge, J. R., Ghods-Sharifi, S., \& Winstanley, C. A., 2008. Cortico-limbicstriatal circuits subserving different forms of cost-benefit decision making. Cognitive, Affective and Behavioral Neuroscience. https://doi.org/10.3758/CABN.8.4.375

Fouquet, C., Petit, G.H., Auffret, A., Gaillard, E., Rovira, C., Mariani, J., Rondi-Reig, L., 2011. Early detection of age-related memory deficits in individual mice. Neurobiol. Aging 32, 1881-1895. https://doi.org/10.1016/j.neurobiolaging.2009.11.001 
Grootendorst, J., Bour, A., Vogel, E., Kelche, C., Sullivan, P.M., Dodart, J.C., Bales, K., Mathis, C., 2005. Human apoE targeted replacement mouse lines: H-apoE4 and h-apoE3 mice differ on spatial memory performance and avoidance behavior. Behav. Brain Res. 159, 114. https://doi.org/10.1016/j.bbr.2004.09.019

Hankosky, E.R., Sherrill, L.K., Ruvola, L.A., Haake, R.M., Kim, T., Hammerslag, L.R., Kougias, D.G., Juraska, J.M., Gulley, J.M., 2017. Effects of $\beta$-hydroxy- $\beta$-methyl butyrate on working memory and cognitive flexibility in an animal model of aging. Nutr. Neurosci. 20, 379-387. https://doi.org/10.1080/1028415X.2016.1145376

Hoeffer, C.A., Tang, W., Wong, H., Santillan, A., Patterson, R.J., Martinez, L.A., Tejada-Simon, M. V., Paylor, R., Hamilton, S.L., Klann, E., 2008. Removal of FKBP12 Enhances mTORRaptor Interactions, LTP, Memory, and Perseverative/Repetitive Behavior. Neuron 60, 832-845. https://doi.org/10.1016/j.neuron.2008.09.037

Jarlier, F., Arleo, A., Petit, G.H., Lefort, J.M., Fouquet, C., Burguière, E., Rondi-Reig, L., 2013. A Navigation Analysis Tool (NAT) to assess spatial behavior in open-field and structured mazes. J. Neurosci. Methods 215, 196-209. https://doi.org/10.1016/j.jneumeth.2013.02.018

Joly, M., Ammersdörfer, S., Schmidtke, D., Zimmermann, E., 2014. Touchscreen-Based Cognitive Tasks Reveal Age-Related Impairment in a Primate Aging Model, the Grey Mouse Lemur (Microcebus murinus). PLoS One 9, e109393. https://doi.org/10.1371/journal.pone.0109393

Knouff, C., Hinsdale, M.E., Mezdour, H., Altenburg, M.K., Watanabe, M., Quarfordt, S.H., Sullivan, P.M., Maeda, N., 1999. Apo E structure determines VLDL clearance and atherosclerosis risk in mice. J. Clin. Invest. 103, 1579-1586. 
https://doi.org/10.1172/JCI6172

Lancaster, C., Tabet, N., Rusted, J., 2017. The Elusive Nature of APOE $\varepsilon 4$ in Mid-adulthood: Understanding the Cognitive Profile. J. Int. Neuropsychol. Soc. 23, 239-253. https://doi.org/10.1017/S1355617716000990

Matzel, L.D., Light, K.R., Wass, C., Colas-Zelin, D., Denman-Brice, A., Waddel, A.C., Kolata, S., 2011. Longitudinal attentional engagement rescues mice from age-related cognitive declines and cognitive inflexibility. Learn. Mem. 18, 345-356. https://doi.org/10.1101//m.2034711

Rawle, M.J., Davis, D., Bendayan, R., Wong, A., Kuh, D., Richards, M., 2018. ApolipoproteinE (Apoe) $\varepsilon 4$ and cognitive decline over the adult life course. Transl. Psychiatry 8, 18. https://doi.org/10.1038/s41398-017-0064-8

Reverte, I., Klein, A.B., Ratner, C., Domingo, J.L., Colomina, M.T., 2012. Behavioral phenotype and BDNF differences related to apoE isoforms and sex in young transgenic mice. Exp. Neurol. 237, 116-125. https://doi.org/10.1016/j.expneurol.2012.06.015

Rodriguez, G.A., Burns, M.P., Weeber, E.J., Rebeck, G.W., 2013. Learning and memory , with reduced dendritic spine density in the medial entorhinal cortex Young APOE4 targeted replacement mice exhibit poor spatial learning and memory, with reduced dendritic spine density in the medial entorhinal. Cortex, 256-266. https://doi.org/10.1101//m.030031.112

Rogers, D.C., Fisher, E.M., Brown, S.D., Peters, J., Hunter, A.J., Martin, J.E., 1997. Behavioral and functional analysis of mouse phenotype: SHIRPA, a proposed protocol for comprehensive phenotype assessment. Mamm. Genome 8, 711-3.

Rondi-Reig, L., Petit, G., Arleo, A., Burguiere, E., 2005. The starmaze: a new paradigm to 
characterize multiple spatial navigation strategies. Nat Neurosci. 8(10), 1292-4

Schoenbaum, G., Nugent, S., Saddoris, M. P., \& Gallagher, M., 2002. Teaching old rats new tricks: Age-related impairments in olfactory reversal learning. Neurobiology of Aging, 23(4), 555-564. https://doi.org/10.1016/S0197-4580(01)00343-8

Stöckel, T., Wunsch, K., Hughes, C.M.L., 2017. Age-Related Decline in Anticipatory Motor Planning and Its Relation to Cognitive and Motor Skill Proficiency. Front. Aging Neurosci. 9, 283. https://doi.org/10.3389/fnagi.2017.00283

Sullivan, P.M., Mezdour, H., Aratani, Y., Knouff, C., Najib, J., Reddick, R.L., Quarfordt, S.H., Maeda, N., 1997. Targeted replacement of the mouse apolipoprotein E gene with the common human APOE3 allele enhances diet-induced hypercholesterolemia and atherosclerosis. J. Biol. Chem. 272, 17972-80.

Trinh, M.A., Kaphzan, H., Wek, R.C., Pierre, P., Cavener, D.R., Klann, E., 2012. Brain-Specific Disruption of the elF2 $\alpha$ Kinase PERK Decreases ATF4 Expression and Impairs Behavioral Flexibility. Cell Rep. 1, 676-688. https://doi.org/10.1016/j.celrep.2012.04.010

Ungar, L., Altmann, A., Greicius, M.D., 2014. Apolipoprotein E, gender, and Alzheimer's disease: An overlooked, but potent and promising interaction. Brain Imaging Behav. 8, 262-273. https://doi.org/10.1007/s11682-013-9272-x

Wetter, S.R., Delis, D.C., Houston, W.S., Jacobson, M.W., Lansing, A., Cobell, K., Salmon, D.P., Bondi, M.W., 2005. Deficits in inhibition and flexibility are associated with the APOE-E4 allele in nondemented older adults. J. Clin. Exp. Neuropsychol. 27, 943-952. https://doi.org/10.1080/13803390490919001

Yang, W., Zhou, X., Ma, T., 2019. Memory Decline and Behavioral Inflexibility in Aged Mice Are Correlated With Dysregulation of Protein Synthesis Capacity. Front. Aging Neurosci. 
11. https://doi.org/10.3389/fnagi.2019.00246

Zamzow, D. R., Elias, V., Shumaker, M., Larson, C., \& Magnusson, K. R., 2013. An increase in the association of GluN2B containing NMDA receptors with membrane scaffolding proteins was related to memory declines during aging. Journal of Neuroscience, 33(30), 12300-12305. https://doi.org/10.1523/JNEUROSCI.0312-13.2013

Zeamer, A., Decamp, E., Clark, K., Schneider, J.S., 2011. Attention, executive functioning and memory in normal aged rhesus monkeys. Behav. Brain Res. 219, 23-30. https://doi.org/10.1016/J.BBR.2010.12.021

Zehnder, A.E., Bläsi, S., Berres, M., Monsch, A.U., Stähelin, H.B., Spiegel, R., 2009. Impact of APOE status on cognitive maintenance in healthy elderly persons. Int. J. Geriatr. Psychiatry 24, 132-141. https://doi.org/10.1002/gps.2080

\section{Figures and Tables}


Day 1: learning

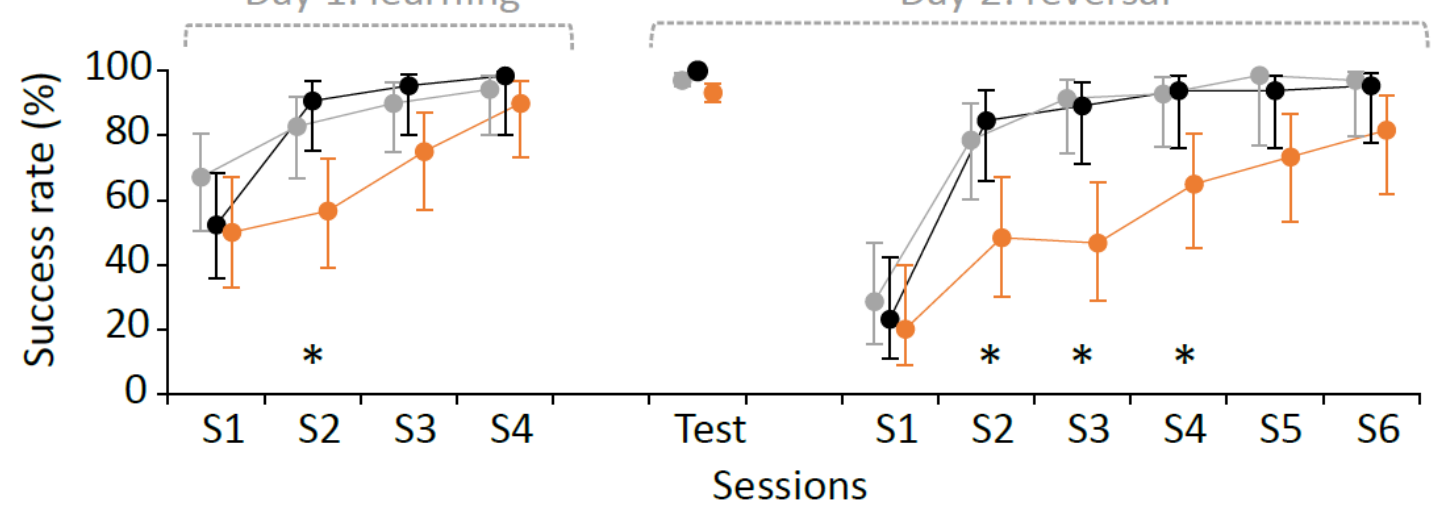

-ApoE3 6m (n=13) -ApoE4 6m (n=12) - C57BL6 6m (n=14)
B

\section{LONGITUDINAL STUDY}

Day 1: learning

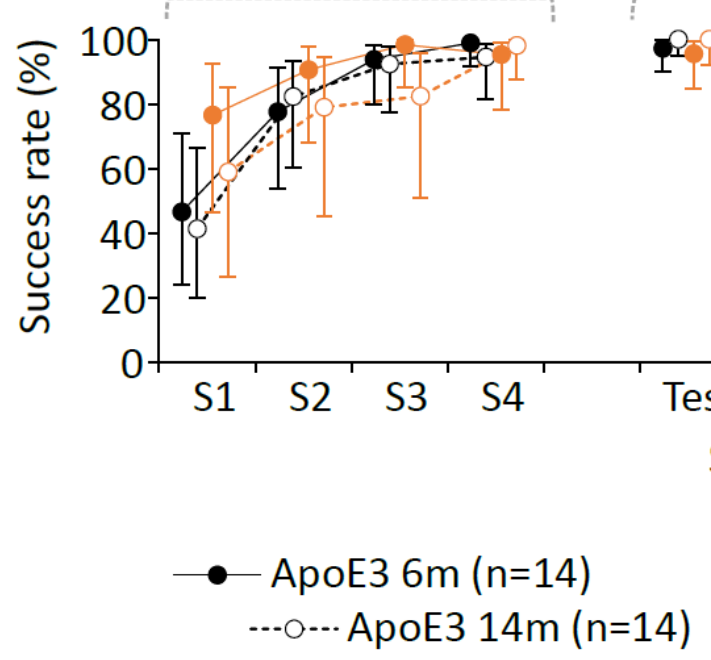

C

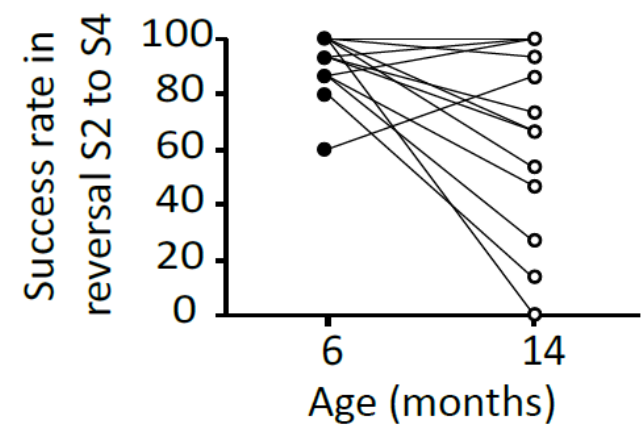

Day 2: reversal

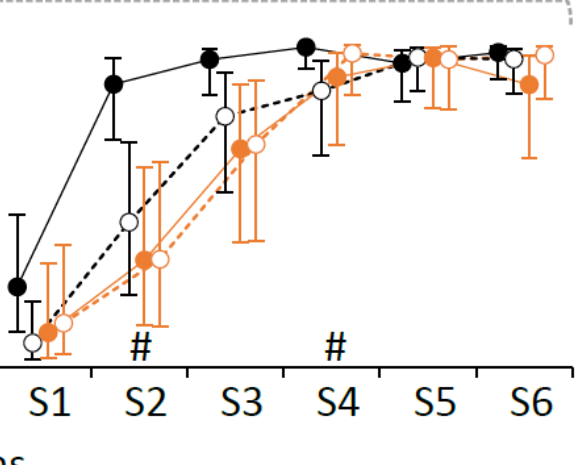

- ApoE4 6m (n=9)

ApoE4 14m ( $n=9)$

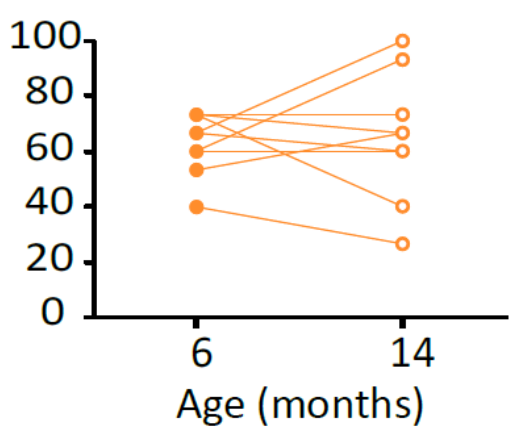


Fig. 1. ApoE4 mice exhibit an early cognitive flexibility deficit.

A. Performances in the aquatic Y-maze of 6 month-old C57BL/6 $(n=14)$, ApoE3 $(n=13)$ and ApoE4 ( $n=12$ ) mice; left S1 to S4: learning sessions on day 1; Test: memory test session performed on the second day to select mice for the reversal test; right S1 to S6: reversal sessions on day 2. Data are expressed as calculated success rate $+/-95 \%$ confidence interval.

*: ApoE4 $<$ ApoE3 \& C57BL/6, p $<0.05$ on a specific session with Student's t test post-hoc followed by Bonferroni-Holm correction for multiplicity after binomial GLMM (Table 1).

B. Comparison of the performances of another group of ApoE3 $(n=14)$ and ApoE4 $(n=9)$ mice in the aquatic Y-maze at 6 (solid lines) and 14 (dotted lines) months old; left S1 to S4: learning sessions on day 1; Test: memory test session performed on the second day to select mice able to do the reversal test; right S1 to S6: reversal sessions on day 2. Data are expressed as calculated success rate +/- 95\% confidence interval. \#: significant difference between performances of ApoE3 at $14 \mathrm{~m}$ and $6 \mathrm{~m}$ on a specific session, with Student's t post-hoc test followed by Bonferroni-Holm correction for multiplicity after binomial GLMM (Table 2).

C. Representation of the evolution between 6 and 14 months of the individual performances of the ApoE3 (left) and ApoE4 (right) mice in the Y-maze reversal sessions S2 to S4. The mean percentage of success in sessions S2, S3 and S4 is presented. 
A

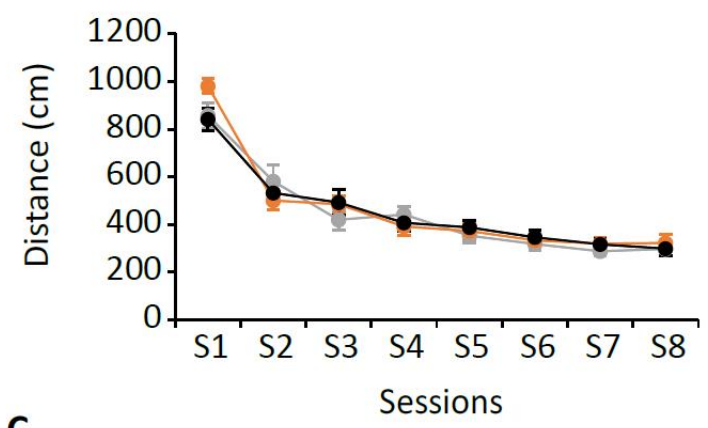

C

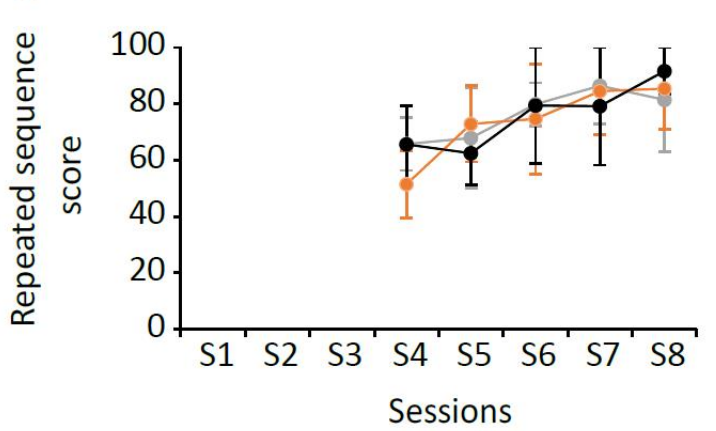

B
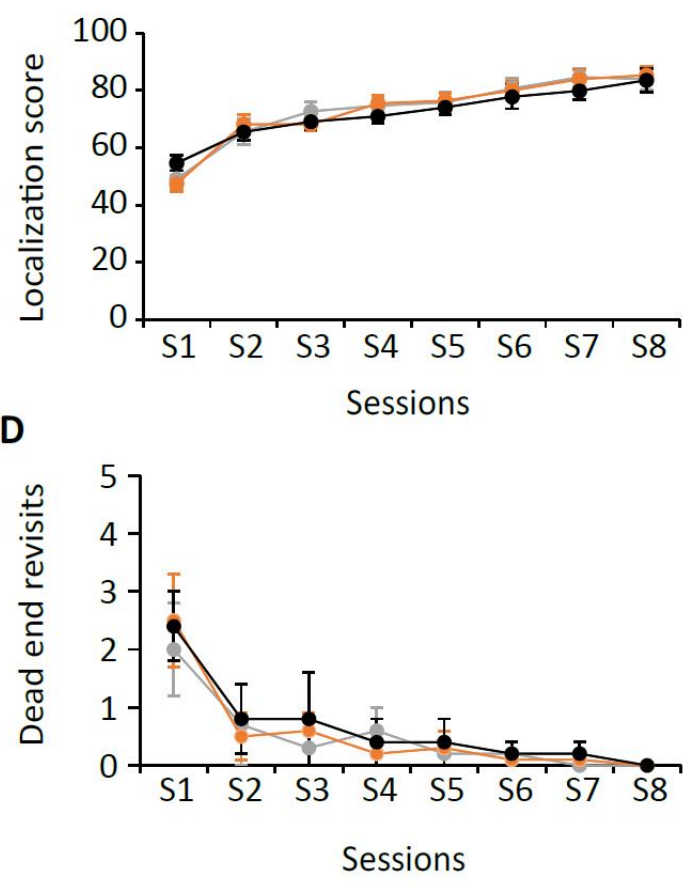

-ApoE3 6m (n=14) -ApoE4 6m (n=14) - C57BL6 6m (n=15)

$E$
$\frac{\pi}{3}$
$\frac{7}{2}$
4
0
0
2
2

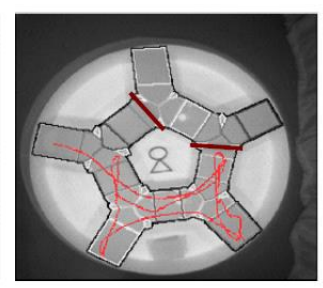

No strategy

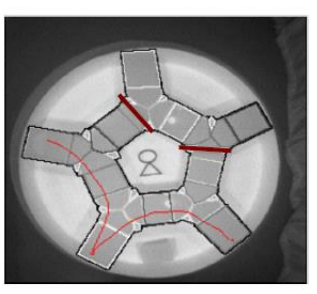

Serial strategy

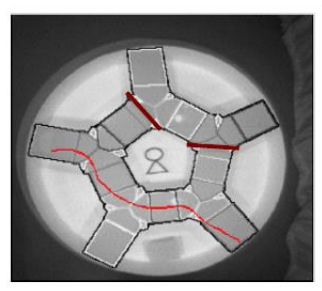

Direct

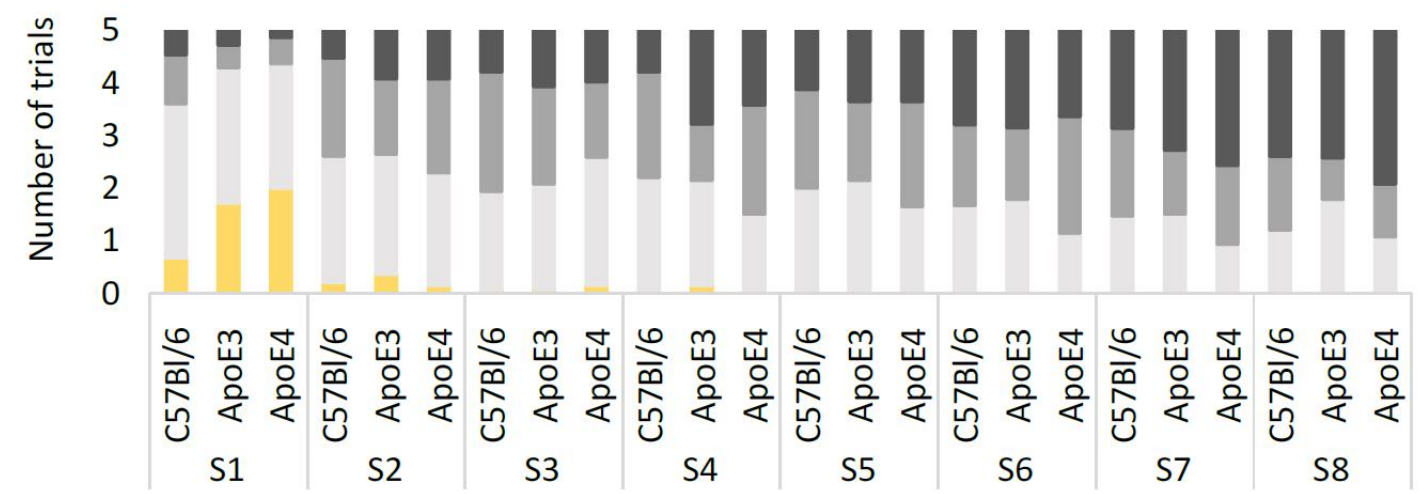


Fig. 2. ApoE4 has no impact on spatial memory in the Starmaze task at 6 months.

Comparison of C57BL/6 $(n=15)$, ApoE3 $(n=14)$ and ApoE4 $(n=14)$ mice at 6 months in the Starmaze. A: Travelled distance, represented as mean +/sem. B: Localization score, represented as mean $+/$ sem. C: Repeated sequence score, represented as median $+/$ mad (median absolute deviation). D: Dead end revisit number, as median +/mad. E: Distribution of trial types by session and genotype. Whatever the score, no significant difference was observed between genotypes (Table 3). 


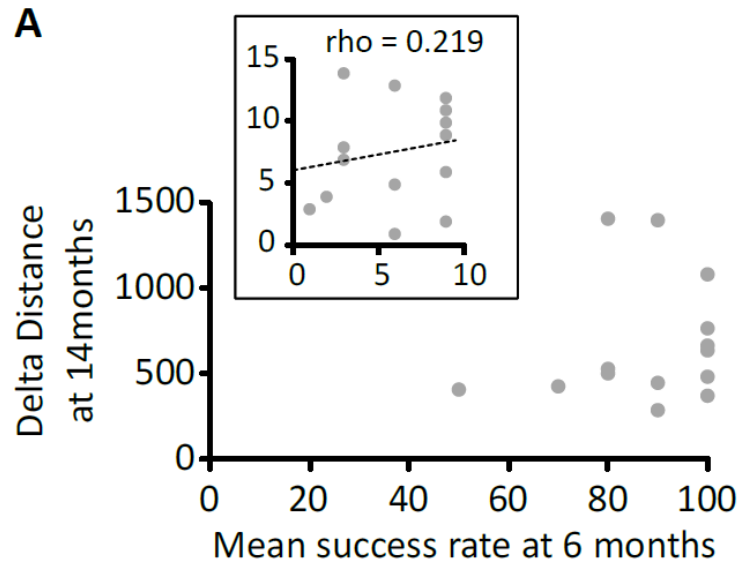

in reversal S2 and S3

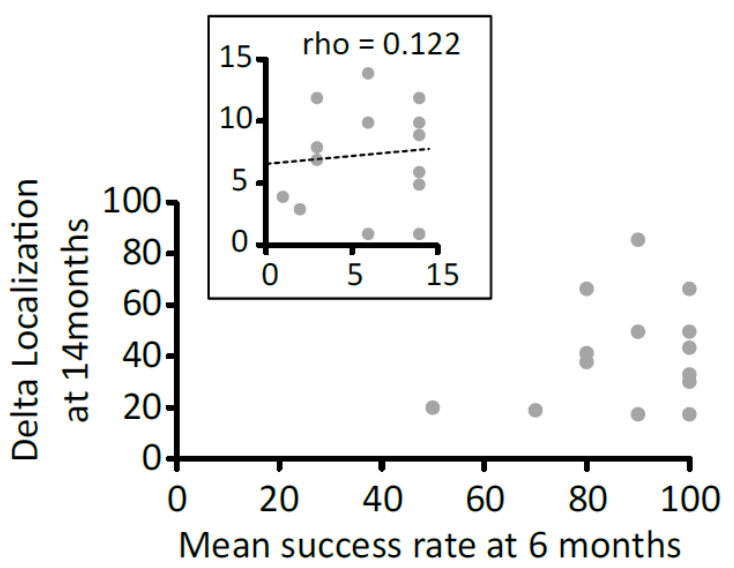

in reversal S2 and S3

- ApoE3 6m \& 14m ( $n=14)$

- ApoE4 6m \& 14 m (n=9)

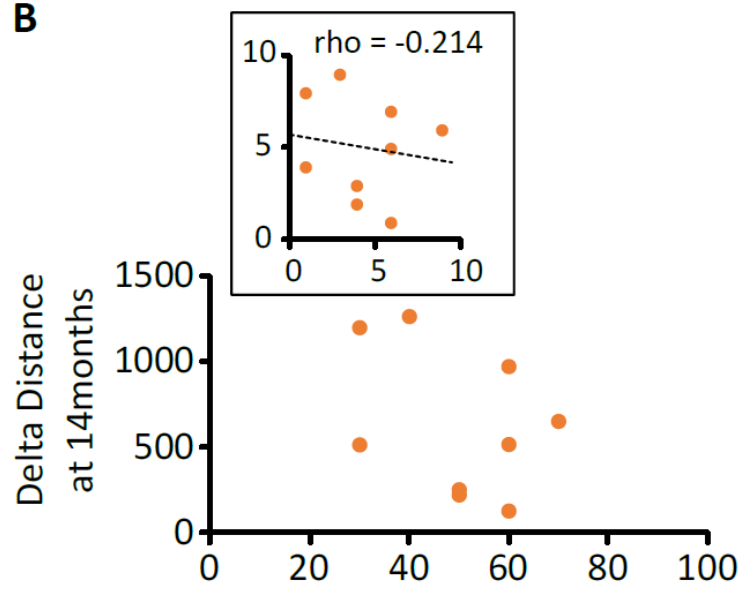

Mean success rate at 6 months in reversal S2 and S3

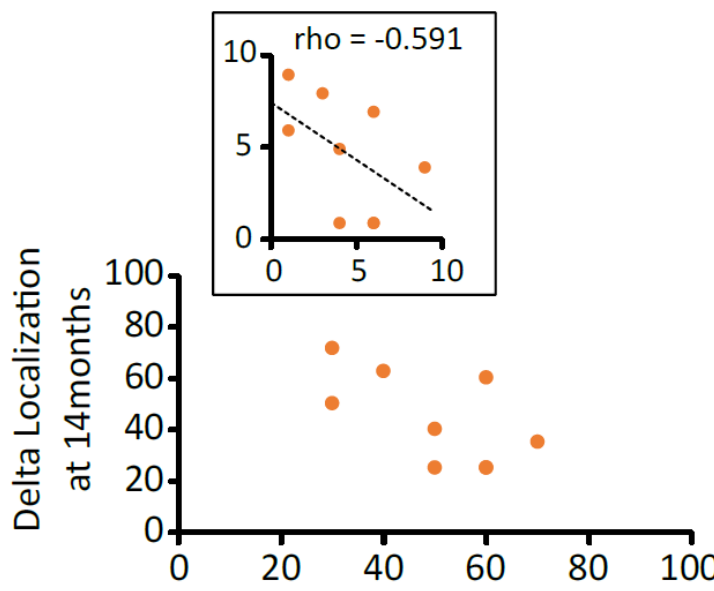

Mean success rate at 6 months in reversal S2 and S3 
Fig. 3. No significant association between flexibility performances at 6 months and learning in the Starmaze at 14 months, in either ApoE3 ( $N=14)$ or ApoE4 $(N=9)$ mice.

Correlation plot of individual performances in the reversal phase of the Y-maze (mean of session S2 and S3) at 6 months old and two learning scores of the Starmaze at 14 months old for ApoE3 (A) and Apoe4 (B) mice. The considered learning scores are the difference between the last and first trials (called delta) of the travelled distance (left) or the localization score (right). The main plots show the raw data and the insets in the top right hand side show the ranked data used to calculate Spearman's correlation (Table 4). 

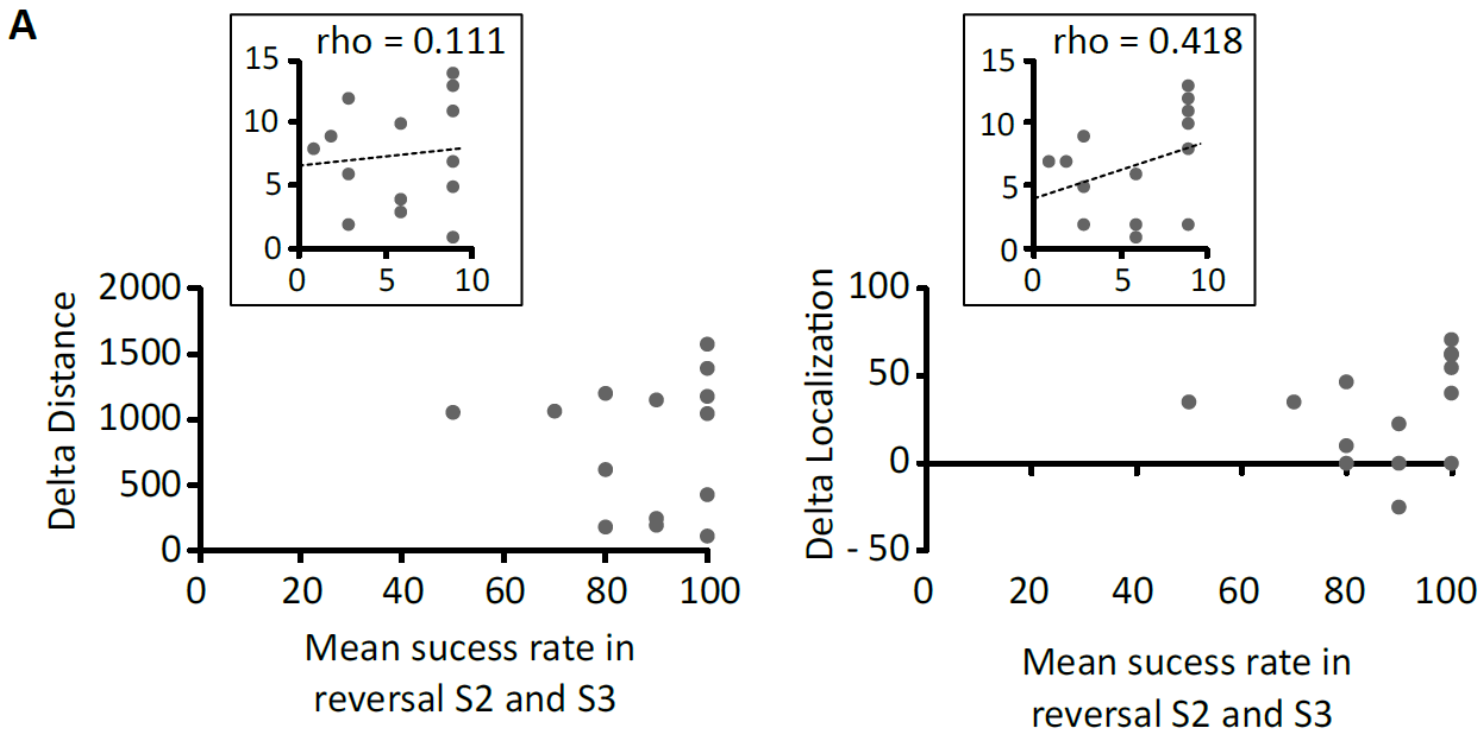

B

- ApoE3 6m (n=14)
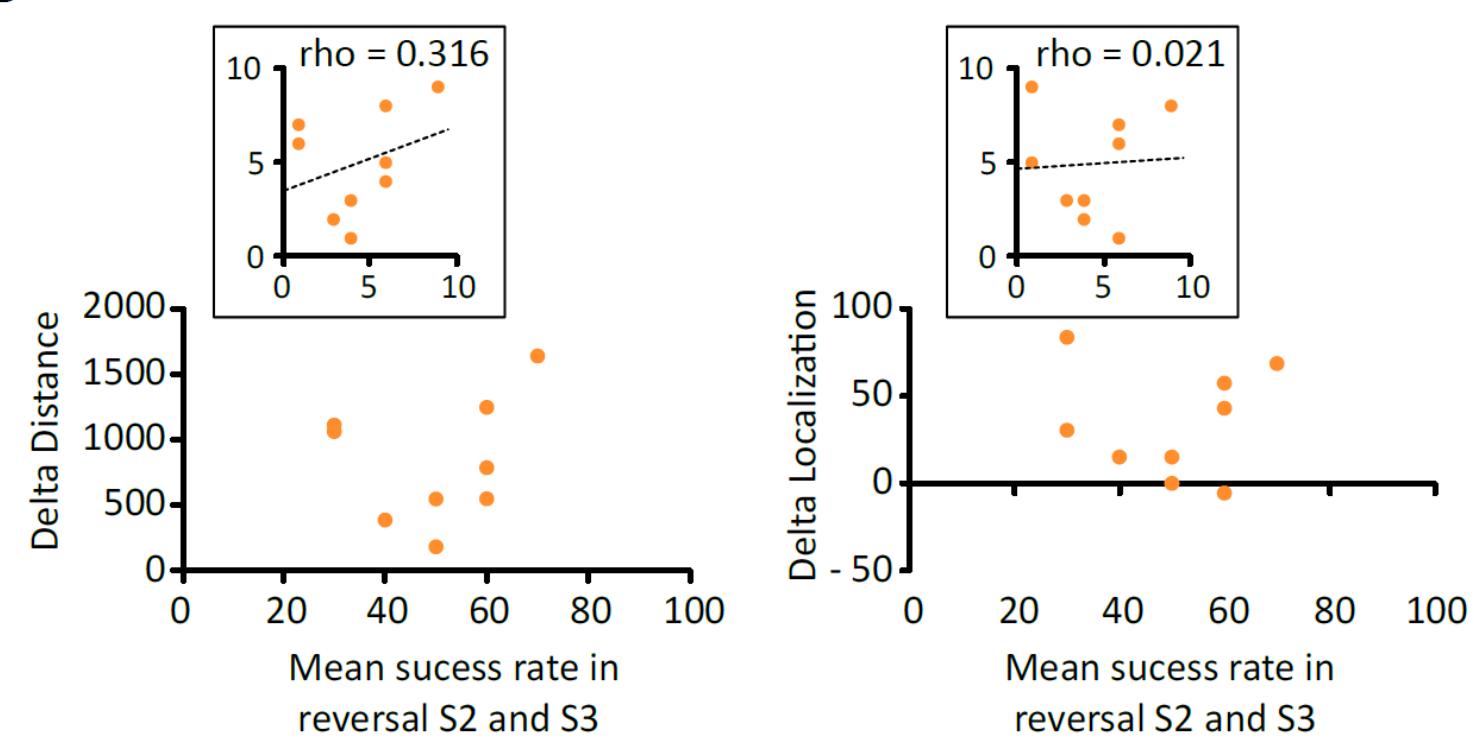

Fig. 4. No significant correlation between flexibility in the $Y$-maze reversal phase and learning in the Starmaze at 6 months in either ApoE3 ( $N=14)$ or ApoE4 ( $N=9)$ mice.

Correlation test was performed between individual performances at 6 months in the reversal phase of the $\mathrm{Y}$-maze (mean of sessions S2 and S3) and in two learning scores of the Starmaze, for ApoE3 (A) and Apoe4 (B) mice. The main plots show the raw data and 
the insets in the top right hand side show the ranked data used to calculate Spearman's correlation (Table 4). 


\section{Tables of statistics performed}

\begin{tabular}{|c|c|c|c|c|c|}
\hline \multicolumn{3}{|c|}{ Learning } & \multicolumn{3}{|c|}{ Reversal } \\
\hline Factor & F value & $P$ value & Factor & F value & $P$ value \\
\hline Genotype & $F(2,52.71)=4.56$ & 0.0149 & Genotype & $F(2,51.17)=6.32$ & 0.0035 \\
\hline Session & $F(3,107.9)=11.51$ & $<0.0001$ & Session & $F(5,189.3)=24.07$ & $<0.0001$ \\
\hline $\begin{array}{l}\text { Genotype } \\
\text { *Session }\end{array}$ & $F(6,112.2)=1.34$ & 0.2454 & $\begin{array}{l}\text { Genotype } \\
\text { * Session }\end{array}$ & $F(10,188.6)=1.36$ & 0.2022 \\
\hline Session & $\begin{array}{c}\text { Group compared } \\
\text { to ApoE4 }\end{array}$ & $\begin{array}{l}\text { Adjusted } \\
\text { p value }\end{array}$ & Session & $\begin{array}{c}\text { Group compared } \\
\text { to ApoE4 }\end{array}$ & $\begin{array}{l}\text { Adjusted } \\
p \text { value }\end{array}$ \\
\hline \multirow{2}{*}{ S1 } & ApoE3 & 0.8533 & \multirow{2}{*}{ S1 } & ApoE3 & 0.9197 \\
\hline & C57BL/6 & 0.3182 & & C57BL/6 & 0.9197 \\
\hline \multirow{2}{*}{ S2 } & ApoE3 & 0.0090 & \multirow{2}{*}{ S2 } & ApoE3 & 0.0154 \\
\hline & C57BL/6 & 0.0237 & & C57BL/6 & 0.0227 \\
\hline \multirow{2}{*}{ S3 } & ApoE3 & 0.0764 & \multirow{2}{*}{ S3 } & ApoE3 & 0.0026 \\
\hline & $\mathrm{C} 57 \mathrm{BL} / 6$ & 0.1151 & & $\mathrm{C} 57 \mathrm{BL} / 6$ & 0.0026 \\
\hline \multirow{2}{*}{ S4 } & ApoE3 & 0.4014 & \multirow{2}{*}{ S4 } & ApoE3 & 0.0374 \\
\hline & C57BL/6 & 0.5175 & & $\mathrm{C} 57 \mathrm{BL} / 6$ & 0.0374 \\
\hline & & & \multirow{2}{*}{ S5 } & ApoE3 & 0.0907 \\
\hline & & & & $\mathrm{C} 57 \mathrm{BL} / 6$ & 0.0907 \\
\hline & & & S6 & ApoE3 & 0.1869 \\
\hline
\end{tabular}




\begin{tabular}{|l|l|l|} 
& C57BL/6 & 0.1869 \\
\hline
\end{tabular}


Table 1. ApoE4 effect at 6 months in the aquatic $Y$-maze.

Top: Analyses of the success rate of 6-month-old ApoE3 $(n=13)$, ApoE4 $(n=12)$ and C57BL/6 ( $n=14$ ) mice during learning (left) and reversal (right) phases. A generalized linear mixed model (GLMM) with binomial distribution was used on raw data with factors Genotype, Session (repeated) and their interaction. Bottom: Post-hoc analysis comparing ApoE4 with ApoE3 or C57BL/6 mice at each session of the Y-maze. Adjusted $p$-values were obtained after the two-way GLMM and Bonferroni-Holm correction for multiplicity. Bold: p-value $<0.05$. 


\begin{tabular}{|c|c|c|c|c|c|}
\hline \multicolumn{3}{|c|}{ Learning } & \multicolumn{3}{|c|}{ Reversal } \\
\hline Factor & F value & P value & Factor & F value & P value \\
\hline Genotype & $F(1,25.26)=0.42$ & 0.5250 & Genotype & $F(1,19.94)=1.85$ & 0.1892 \\
\hline Age & $F(1,25.03)=1.58$ & 0.2197 & Age & $F(1,21.56)=1.01$ & 0.3252 \\
\hline Session & $F(3,168)=22.35$ & $<0.0001$ & Session & $F(5,99.63)=47.56$ & $<0.0001$ \\
\hline Genotype*Age & $F(1,25.03)=0.16$ & 0.6921 & Genotype*Age & $F(1,21.56)=4.38$ & 0.0483 \\
\hline $\begin{array}{l}\text { Genotype } \\
\text { *Session }\end{array}$ & $F(3,168)=0.74$ & 0.5274 & $\begin{array}{l}\text { Genotype } \\
\text { *Session }\end{array}$ & $F(5,99.63)=1.67$ & 0.1479 \\
\hline Age*Session & $F(3,168)=0.52$ & 0.6699 & Age* Session & $F(5,93.78)=1.25$ & 0.2927 \\
\hline $\begin{array}{c}\text { Genotype } \\
\text { *Age*Session }\end{array}$ & $F(3,168)=1.93$ & 0.1267 & $\begin{array}{c}\text { Genotype } \\
\text { *Age *Session }^{*} \text { * }\end{array}$ & $F(5,93.78)=1.40$ & 0.2320 \\
\hline
\end{tabular}

\begin{tabular}{|c|c|c|c|c|}
\hline \multirow{2}{*}{ Reversal } & \multicolumn{2}{|c|}{ Session by session analysis } \\
\cline { 2 - 5 } & \multicolumn{2}{|c|}{$\begin{array}{c}\text { Effect of age (6 vs. 14 months) } \\
\text { (adjusted p-value) }\end{array}$} & \multicolumn{2}{c|}{$\begin{array}{c}\text { Effect of genotype } \\
\text { (ApoE3 vs. ApoE4) (p-value) }\end{array}$} \\
\hline Session & in ApoE3 & in ApoE4 & 0.0939 \\
\hline S1 & 0.1131 & 0.7663 & 0.0001 \\
\hline S2 & $\mathbf{0 . 0 0 8 1}$ & 0.9881 & 0.0096 \\
\hline S3 & 0.0710 & 0.9378 & & 0.1298 \\
\hline S4 & 0.0342 & 0.2320 & & 0.5124 \\
\hline S6 & 1.000 & 1.000 & & 0.1884 \\
\hline
\end{tabular}


Table 2. Aging effect in the aquatic Y-maze.

Analysis of the success rate of ApoE3 $(n=13)$ and ApoE4 $(n=12)$ mice at 6 and 14 months. Top: Separate binomial generalized linear mixed models (GLMM) with factors Genotype, Session (repeated), Age (repeated) and their interactions were applied on the success rate of the learning (left) and the reversal (right) phase. Bottom: Session by session comparisons on success rate in the reversal phase of the Y-maze. Effect of age on success rate in the Y-maze reversal phase was estimated after the significant age*genotype interaction effect found with the binomial GLMM and was followed by Bonferroni-Holm correction for multiplicity in ApoE3 and ApoE4 mice at each session. The comparison of Apoe3 vs. Apoe4 mice at 6 months allows to confirm that the longitudinal group reproduces the results of the 6 months group. Bold: $p$-value $<0.05$. 


\begin{tabular}{|c|c|c|c|c|}
\hline Statistical model & Parameters & Factor & F value & $P$ value \\
\hline \multirow{6}{*}{$\begin{array}{l}\text { Two-way } \\
\text { ANOVA }\end{array}$} & \multirow{3}{*}{$\begin{array}{l}\text { Travelled } \\
\text { distance }\end{array}$} & Genotype & $F(2,85.01)=0.40$ & 0.6739 \\
\hline & & Session & $F(7,266.2)=52.12$ & $<0.0001$ \\
\hline & & Genotype*Session & $F(14,277.5)=0.97$ & 0.4855 \\
\hline & \multirow{3}{*}{$\begin{array}{l}\text { Localization } \\
\text { score }\end{array}$} & Genotype & $F(2,75.6)=0.24$ & 0.7859 \\
\hline & & Session & $F(7,264)=27.90$ & $<0.0001$ \\
\hline & & Genotype*Session & $F(14,275)=0.59$ & 0.8744 \\
\hline \multirow{6}{*}{$\begin{array}{c}\text { Two-way } \\
\text { ANOVA-type }\end{array}$} & \multirow{3}{*}{$\begin{array}{c}\text { Repeated } \\
\text { sequence score }\end{array}$} & Genotype & $F(2,52.3)=0.20$ & 0.8163 \\
\hline & & Session & $F(3.39,195)=6.60$ & $<0.0001$ \\
\hline & & Genotype*Session & $F(6.79,195)=0.86$ & 0.5354 \\
\hline & \multirow{3}{*}{$\begin{array}{c}\text { Dead end revisit } \\
\text { number }\end{array}$} & Genotype & $F(2,91.1)=0.86$ & 0.4265 \\
\hline & & Session & $F(6.48,319)=27.34$ & $<0.0001$ \\
\hline & & Genotype*Session & $F(13,319)=0.65$ & 0.8128 \\
\hline \multirow{9}{*}{$\begin{array}{c}\text { Two-way } \\
\text { binomial } \\
\text { GLMM }\end{array}$} & \multirow{3}{*}{ Direct strategy } & Genotype & $F(2,40)=0.24$ & 0.7857 \\
\hline & & Session & $F(7,280)=11.76$ & $<0.0001$ \\
\hline & & Genotype*Session & $F(14,280)=0.55$ & 0.9020 \\
\hline & \multirow{3}{*}{ Serial strategy } & Genotype & $F(2,40)=1.09$ & 0.3446 \\
\hline & & Session & $F(7,280)=3.51$ & 0.0013 \\
\hline & & Genotype*Session & $F(14,280)=0.71$ & 0.7672 \\
\hline & \multirow{3}{*}{ No Strategy } & Genotype & $F(2,40)=1.13$ & 0.3318 \\
\hline & & Session & $F(7,280)=2.77$ & 0.0086 \\
\hline & & Genotype*Session & $F(14,280)=0.48$ & 0.9452 \\
\hline
\end{tabular}




\begin{tabular}{|c|c|c|c|c|c|c|c|c|c|}
\hline & & \multicolumn{6}{|c|}{ Session by session analysis of search strategies (p-values) } \\
\hline \multirow{2}{*}{ Score } & $\begin{array}{c}\text { Group vs. } \\
\text { ApoE4 }\end{array}$ & S1 & S2 & S3 & S4 & S5 & S6 & S7 & S8 \\
\hline $\begin{array}{c}\text { Direct } \\
\text { strategy }\end{array}$ & ApoE3 & 0.5874 & 1.0000 & 0.8901 & 0.5494 & 1.0000 & 0.7247 & 0.6541 & 0.4327 \\
\cline { 2 - 10 } & C57bl6 & 0.3116 & 0.3759 & 0.6780 & 0.2324 & 0.6798 & 0.7920 & 0.2598 & 0.3993 \\
\hline \multirow{2}{*}{$\begin{array}{c}\text { Serial } \\
\text { strategy }\end{array}$} & ApoE3 & 0.8592 & 0.5819 & 0.5115 & 0.1262 & 0.4510 & 0.2010 & 0.6436 & 0.6872 \\
\hline & C57bl6 & 0.3735 & 0.9019 & 0.2072 & 0.9151 & 0.8408 & 0.3040 & 0.7929 & 0.4947 \\
\hline $\begin{array}{c}\text { No } \\
\text { Strategy }\end{array}$ & ApoE3 & 0.7253 & 0.8136 & 0.4800 & 0.3915 & 0.3992 & 0.2502 & 0.2786 & 0.1991 \\
\cline { 2 - 10 } & C57bl6 & 0.3370 & 0.6669 & 0.3450 & 0.2299 & 0.5368 & 0.3346 & 0.2981 & 0.7982 \\
\hline
\end{tabular}

Table 3. Comparison of 6-month-old ApoE4 $(N=14)$, ApoE3 $(N=14)$ and C57BI6 $(N=15)$ mice in the Starmaze.

Top: Effects of factors Genotype, Session (repeated) and their interaction. Bottom: Session by session comparison of the percentage of trials showing Direct, Serial or No strategy in APoE4 vs. ApoE3 and C57BI6 mice. Bold: $p<0.05$ and $p<0.1$ for interaction. 


\begin{tabular}{|c|c|c|c|c|}
\hline Tested association & Genotype & learning score & rho & p \\
\hline \multirow{4}{*}{$\begin{array}{c}6 \mathrm{~m} \text { flexibility } \\
\text { vs. } \\
14 \mathrm{~m} \text { learning }\end{array}$} & \multirow{2}{*}{ ApoE3 } & Distance & 0.219 & 0.4289 \\
\hline & & Localization & 0.122 & 0.6609 \\
\hline & \multirow{2}{*}{ ApoE4 } & Distance & -0.214 & 0.5455 \\
\hline & & Localization & -0.591 & 0.0944 \\
\hline \multirow{4}{*}{$\begin{array}{c}6 \mathrm{~m} \text { flexibility } \\
\text { vs. } \\
6 \mathrm{~m} \text { learning }\end{array}$} & \multirow{2}{*}{ ApoE3 } & Distance & 0.111 & 0.6894 \\
\hline & & Localization & 0.418 & 0.1317 \\
\hline & \multirow{2}{*}{ ApoE4 } & Distance & 0.316 & 0.3709 \\
\hline & & Localization & 0.021 & 0.9516 \\
\hline
\end{tabular}

Table 4. Correlation tests between flexibility and learning indexes.

Spearman's rank correlation performed separately on ApoE3 $(n=14)$ and ApoE4 $(n=9)$ mice. 


\section{Supplementary Material and methods: tests included in}

\section{SHIRPA protocol}

Before cognitive tests, mice perform a short battery of sensorimotor tests inspired by the SHIRPA protocol created by Rogers et al. (1997). This battery of tests takes place over 5 days and is used to ensure that the possible deficits observed during cognitive tests are not related to sensorimotor dysfunctions that could affect mouse behavior. The performed tests and the sensorimotor capacities they evaluate are presented below.

\section{General observation}

The mouse is placed in an empty cage and observed for 2 minutes to determine its general condition. The absence of normal behaviors such as grooming, sniffing, rearing, exploration/movement around the entire cage, as well as the appearance of abnormal behaviors such as freezing during more than 5 seconds, more than 3 urinations or defecations, wild running, and/or jumping can be the sign of an anxious state. If a mouse demonstrates one or more of these abnormal behaviors, its behavior in the following tests will be specifically monitored to determine whether it should be excluded from the study.

At the end of these 2 minutes of observation, a record is made of several physical characteristics (weight, presence of whiskers, piloerection) as well as specific reflexes (response to whisker and ear touch using a cotton swab, blinking when the cotton swab is approached to the eye). An absence of eyelid closure reflex may indicate a vision alteration, and is therefore an exclusion criterion. 


\section{Elevated plus maze}

This test consists of a $41 \mathrm{~cm}$ high elevated cross where two opposite arms are surrounded by walls (closed arms) while the other two are not (open arms). The mouse is placed in the center of the elevated plus maze and for 5 minutes the number of entries and the time spent in each type of arm is recorded manually by the experimenter. The mouse is considered to have entered an arm when it has all four legs in that arm. This test evaluates the anxiety of the mouse by comparing the percentage of time spent in open arms to the percentage of time spent in closed arms. Anxious mice spend more time in the closed arms.

\section{Open field}

The mouse is placed in the center of a $45 \mathrm{~cm}$ square arena in which it is allowed to freely move for 10 minutes. The arena is made of grey opaque plexiglass. The brightness in the arena is between 80 (along the edges) and 100 (in the center) lux. The movements of the mouse are recorded by a camera connected to a computer with the SMART tracking software (Bioseb). This test is used to evaluate the mouse's locomotor abilities through the distance covered.

\section{Unstable platform}

This test consists of a $9 \mathrm{~cm}$ circular platform fixed on an $80 \mathrm{~cm}$ vertical rod. This platform tilts in all directions depending on the position of the mouse. A trial lasts a maximum of 3 minutes. Each mouse performs 3 trials. If the mouse falls before 20 seconds, it is immediately placed back on the platform. If it falls again before the end of the 3 minutes, the time of first fall is manually recorded. This test is used to evaluate the static balance of the mouse. 


\section{Horizontal rod}

The horizontal rod is a fixed cylinder $3 \mathrm{~cm}$ in diameter and $52 \mathrm{~cm}$ long. The mouse is placed on the rod and allowed to explore it for 3 minutes. The distance covered is measured using SMART software (Bioseb). This test is used to evaluate the dynamic balance of mice.

\section{6. $\quad$ Accelerated Rotarod}

The rotarod is a device composed of a rotating horizontal cylinder. After a preliminary session to train the mouse to hold the cylinder for 30 seconds at a constant speed of 4 rotations per minute (rpm), the rotarod is set in the accelerated version. A trial lasts a maximum of 5 minutes during which the speed gradually increases from 4 to 40 rpm, with an increase of 1 rpm every 8 seconds. Each mouse performs 3 trials. The holding time and the rotational speed of the cylinder at the time of the fall are recorded. This test is used to evaluate the motor coordination of the mouse.A.2. Aquatic Y-maze.

On the first day, mice undergo 4 sessions of 5 trials. The 5 trials are performed in a row, and two sessions are separated by 7 to 10 minutes. In each trial, the mouse is placed at the end of the starting arm. It has a maximum of 20 seconds to get to the intersection and make a choice, otherwise it is picked up and returned to the starting point for the next trial. If the choice is incorrect, a door locks the mouse in the arm without a platform for 10 to 15 seconds, before the mouse is picked up and returned to the starting point for the next trial. If the choice is correct, the mouse is allowed to reach the platform. It is then picked up directly from it and placed back at the starting point. At the end of each session, the mouse is placed in a cage containing a towel for drying while waiting for the next session. The next day, the mouse performs a first memory test 
session, with the platform located on the same side as the day before. If the mouse succeeds in at least 4 out of 5 trials, it then performs 6 reversal sessions. The protocol is the same as during the learning phase except that the platform is moved to the other side of the $Y$ (stimulus-reward contingency reversal).

\section{Statistical analysis}

For the tests in the SHIRPA protocol (Table S1), the elevated plus maze, the open field distance, the horizontal rod and the accelerated time parameters, a Kruskal Wallis test was performed. For the unstable platform, a Logrank test was performed followed by Dunnett-Hsu adjustment in order to compare ApoE4 and C57BL/6 to ApoE3 group. The significance level was set at 5\%, except for the interaction for which the significance level was set at $10 \%$. 


\begin{tabular}{|c|c|c|c|c|c|}
\hline Test & Parameter & Group & Median +/- MAD & Statistical test & $P$ value \\
\hline \multirow{3}{*}{$\begin{array}{c}\text { Elevated Plus } \\
\text { Maze }\end{array}$} & \multirow{3}{*}{$\begin{array}{l}\text { Percent time } \\
\text { in open arms }\end{array}$} & C57BL/6 & $29.9+/-14.3$ & \multirow{3}{*}{ Kruskal Wallis } & \multirow{3}{*}{0.1869} \\
\hline & & ApoE3 & $34.9+/-10.4$ & & \\
\hline & & ApoE4 & $58.0+/-21.5$ & & \\
\hline \multirow{3}{*}{ Open Field } & \multirow{3}{*}{$\begin{array}{l}\text { Travelled distance } \\
\qquad(\mathrm{cm})\end{array}$} & $\mathrm{C} 57 \mathrm{BL} / 6$ & $2733.9+/-489.7$ & \multirow{3}{*}{ Kruskal Wallis } & \multirow{3}{*}{0.0389} \\
\hline & & ApoE3 & $2263.2+/-445.0$ & & \\
\hline & & ApoE4 & $2454.9+/-567.6$ & & \\
\hline \multirow{3}{*}{ Horizontal Rod } & \multirow{3}{*}{$\begin{array}{l}\text { Travelled distance } \\
\qquad(\mathrm{cm}) \\
\text { mean of } 3 \text { trials }\end{array}$} & $\mathrm{C} 57 \mathrm{BL} / 6$ & $82.3+/-26$ & \multirow{3}{*}{ Kruskal Wallis } & \multirow{3}{*}{0.3159} \\
\hline & & ApoE3 & $91.0+/-24.9$ & & \\
\hline & & ApoE4 & $85.6+/-23.8$ & & \\
\hline \multirow{3}{*}{$\begin{array}{l}\text { Unstable } \\
\text { Platform }\end{array}$} & \multirow{3}{*}{$\begin{array}{l}\text { Time before to fall } \\
\text { (s) } \\
\text { mean of } 3 \text { trials }\end{array}$} & C57BL/6 & $128.3+/-51.7$ & \multirow{3}{*}{ Logrank } & \multirow{3}{*}{0.407} \\
\hline & & ApoE3 & $147.2+/-32.8$ & & \\
\hline & & ApoE4 & $124.0+/-53.2$ & & \\
\hline \multirow{3}{*}{$\begin{array}{c}\text { Accelerated } \\
\text { Rotarod }\end{array}$} & \multirow{3}{*}{$\begin{array}{l}\text { Time before to fall } \\
\text { (s) } \\
\text { mean of } 3 \text { trials }\end{array}$} & $\mathrm{C} 57 \mathrm{BL} / 6$ & $33.7+/-9.7$ & \multirow{3}{*}{ Kruskal Wallis } & \multirow{3}{*}{0.1720} \\
\hline & & ApoE3 & $27.8+/-6.2$ & & \\
\hline & & ApoE4 & $27.2+/-7.8$ & & \\
\hline
\end{tabular}

Table S1. Comparison of 6-month-old ApoE3 $(n=14)$, ApoE4 $(n=14)$ and C57BL/6 $(n=15)$ mice in SHIRPA protocol.

MAD: median absolute deviation. Bold: $p$-value $<0.05$. 